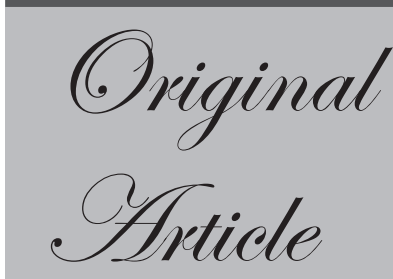

Department of GI Surgery \& Liver

Transplant, GB Pant Hospital \& MAM

College, New Delhi 110002

Correspondence: Anil K Agarwal

Email:aka.hpb@gmail.com

anilagarwal@outlook.com

\section{Laparoscopic resectional surgery for hydatid disease of the liver}

\author{
Nikhil Gupta, Amit Javed, MN Saravanan, \\ Raja Kalayarasan, Sunil Puri, Anil K Agarwal
}

ABSTRACT

Background: Resectional procedures for hydatid cyst are associated with decreased cavity related complications and recurrence rate. In contrast to laparoscopic drainage procedures, the evidence for laparoscopic resection of hydatid cyst is limited in the literature.

Methods: A retrospective analysis of a prospectively maintained database of patients with hydatid cyst managed between January 2006 and December 2011. Patients planned for laparoscopic resectional surgery for hydatid cyst were included in the analysis. Clinical features, cyst location, size, type of cyst, operative parameters, reasons for conversion and postoperative recurrence rate were analysed.

Results: Of the 56 patients with hydatid cyst managed surgically during the study period, 35 patients underwent resectional procedures. Fourteen patients planned for the laparoscopic resectional procedure were included in the analysis. Abdominal pain was the most common presenting symptom and the majority (10/14) had Gharbi type II/III cyst. Laparoscopic cystopericystectomy $(\mathrm{n}=5)$ was the commonly performed procedure followed by laparoscopic left lateral hepatectomy $(n=4)$. The mean (range) intra-operative blood loss and the mean (range) operating time was 111.1 (50-250) $\mathrm{mL}$ and 243.3 (180-300) minutes respectively. Five patients required conversion to open surgery in view of bleeding or posterosuperior location of the cyst. None of the patients had intra-operative spillage of cyst contents. At a median (range) follow-up of 54 (21-86) months, none of the patients had a local or extrahepatic recurrence.

Conclusions: Laparoscopic resectional procedure for hydatid cyst of the liver is technically feasible with excellent perioperative and long-term outcomes. Inability to perform a laparoscopic resectional procedure should not be used as an argument to perform laparoscopic drainage.

KEYWORDS: Hydatid disease, Cystopericystectomy, Echinococcosis. 


\section{Introduction}

Echinococcosis is a significant health problem especially in Eastern Europe, Mediterranean countries, South America and Far East including India. ${ }^{1}$ The disease results from infection with Echinococcus granulosus and manifests as hydatid cysts most commonly in the liver and lungs. ${ }^{2}$ The disease, although asymptomatic in many, may have variable clinical behaviour resulting from mass effect or cyst complications like infection or rupture. Surgery has traditionally been the treatment of choice and includes both conservative operations (drainage) and radical resections (cystopericystectomy, formal hepatectomy). Conservative surgical procedures, both laparoscopic and open, are more popular but have a higher risk of recurrence. Resectional procedures are associated with decreased incidence of postoperative bile leaks, cavity related complications and decreased risk of recurrence and is thus preferred over conservative surgery wherever feasible. ${ }^{3,4,5}$ With the advent of laparoscopic surgery and percutaneous techniques like percutaneous aspiration, injection and re-aspiration (PAIR); and PAIR with catheter drainage (PAIR-D), there has been a change in the treatment strategy for this disease with a resurgence of drainage procedures. Although these have the advantages of being minimally invasive and are technically easier to perform, they still have the same drawbacks of recurrence and cavity-related problems as open drainage procedures. The reports on laparoscopic resectional surgery for hydatid cyst are limited as it is technically challenging (compared to a laparoscopic drainage) and requires greater expertise. In a previous publication, we have described an algorithmic approach for the management of this disease incorporating the various available treatment options. ${ }^{5}$ The present article focuses on the technique, feasibility, and outcomes of performing cystopericystectomy and formal liver resection laparoscopically for hydatid disease of the liver.

\section{Methods}

We performed a retrospective review of all patients who underwent treatment for hydatid cyst of liver at our center between January 2006 and December 2011.

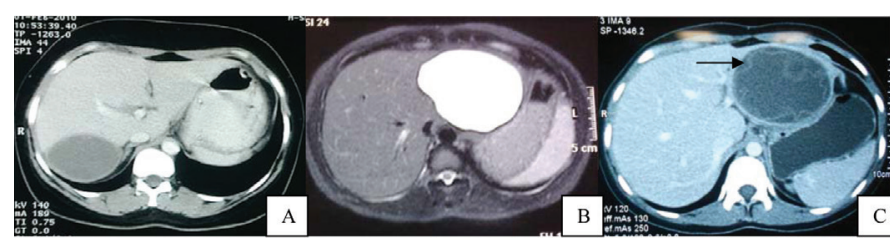

Figure 1: Radiological imaging of hydatid cysts of the liver best suited for laparoscopic radical resections. (A): CECT abdomen showing a hydatid cyst located in segment VI of the liver suited for laparoscopic cystopericystectomy; (B,C): MRI and CECT of the abdomen showing a hydatid cyst occupying whole of left lateral segment suited for left lateral hepatectomy (black arrow in Fig 1C shows hydatid membranes)

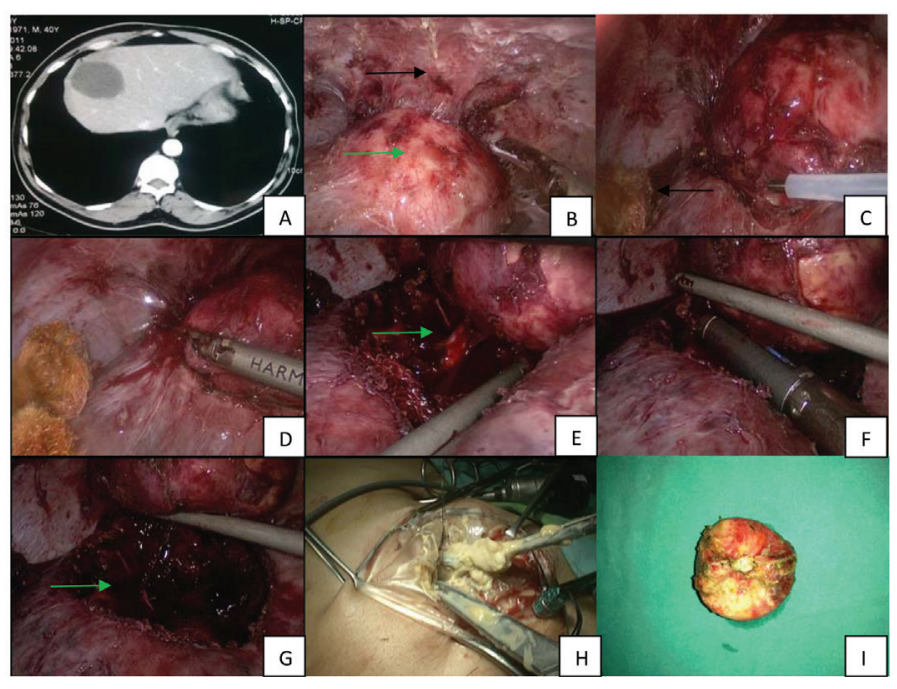

Figure 2: Surgical steps for performing a laparoscopic cystopericystectomy (A): CECT abdomen depicting a hydatid cyst in segment VIII of the liver; (B): Hydatid cyst arising from segment VIII of the liver (green arrow), black arrow depicts the diaphragm; (C): Pericystectomy being performed using a cavitron ultrasonic aspirator, black arrow denotes a betadine soaked gauze placed adjacent to the field of dissection; (D): Pericystectomy being performed using a harmonic scalpel; (E): Hydatid cyst almost completely dissected from the liver, green arrow depicts a biliovascular pedicle entering the cyst; (F): Bilovascular pedicle being stapled using an endo-GIA stapler; (G): Residual cavity after complete cystopericystectomy with no bile staining; $(\mathrm{H})$ : The cyst and contents being evacuated from a small umbilical incision, (note a plastic barrier around the umbilical port to prevent contamination); (I): Excised hydatid cyst (inset depicts the final surgical scar at 3 months followup) 
The data was collected from a prospectively maintained liver disease database and analyzed. All patients with suspected hydatid cyst of the liver were evaluated with an ELISA for Echinococcus antibodies, ultrasound and contrast-enhanced CT scan of the abdomen. The cyst morphology on ultrasound was classified according to the Gharbi classification. ${ }^{6}$ An MRCP was added in patients when indicated. In addition, all patients underwent routine hematological and biochemical investigations and received a course of albendazole preoperatively as per the WHO protocol. ${ }^{7}$

For the purpose of this analysis, only patients who were taken up for laparoscopic cystopericystectomy or laparoscopic formal liver resection were considered. A cystopericystectomy was done for small peripheral cysts located in anterior segments, and a formal left lateral segmentectomy was performed for cysts occupying most of the left lateral lobe. Laparoscopic cystopericystectomy was preferred but when a whole lobe was replaced with hydatid cyst, a formal liver resection was performed. Figure 1 depicts cysts which were found to be amenable for laparoscopic radical resections.

All patients were followed up with serial ultrasounds every third month for the first year. A contrastenhanced CT scan of the whole abdomen was done yearly to rule out any other site of extrahepatic recurrence.

\section{Surgical Technique}

Cystopericystectomy (Figure 2): The cyst was isolated by using povidone-iodine (10\%) soaked surgical gauzes. A plane was created between the pericyst and the normal liver parenchyma using a cavitron ultrasonic surgical aspirator $\left(\mathrm{CUSA}^{\mathrm{TM}}\right)$. Further dissection of the cyst was performed using a combination of ultrasonic aspirator (CUSA ${ }^{\mathrm{TM}}$; Valleylab, Boulder, Colorado, USA), Harmonic Scalpel $^{\mathrm{TM}}$ (Ethicon Endosurgery, Cincinnati, Ohio, USA) and electrocautery. Hem-o-lok clips ${ }^{\circledR}$ (Weck Closure System, Research Triangle Park, North Carolina, USA) or Endo GIA staplers/clips/ligatures were used to secure the bilio-vascular pedicles. The excised cyst was removed using an endobag to avoid any intra-peritoneal spillage. The endobag was delivered through one of the abdominal ports, the cyst was opened (within the bag), all

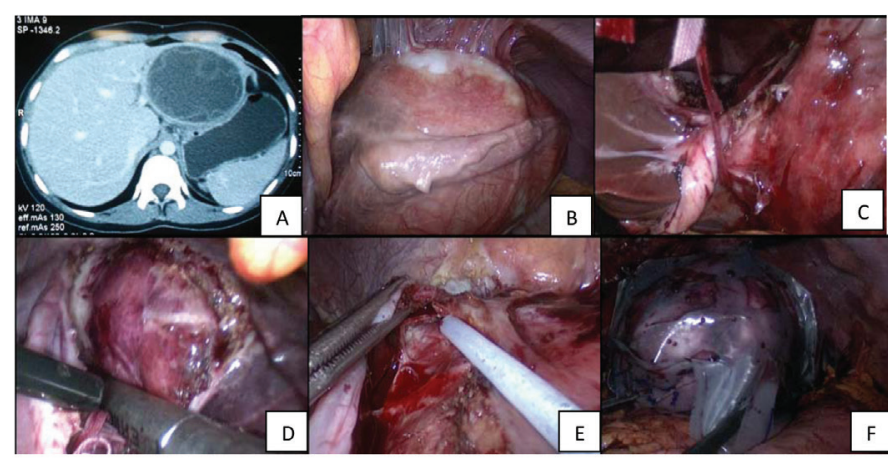

Figure 3: Surgical steps for performing a laparoscopic left lateral hepatectomy for a hydatid cyst localized to the left lateral segments of the liver (A): CT scan imaging of a hydatid cyst occupying the left lateral segment of the liver; (B): Intraoperative photograph of the hydatid cyst to the left of the falciform ligament with pericystic adhesions; (C,D): Inflow to the left lateral segment looped and staple divided; (E): Liver parenchymal transaction with CUSA; (F): Left lateral hepatectomy specimen removed in an eno-bag.

liquid contents were suctioned out and the daughter cysts were removed. This limited the size of the incision. The resection surface of the liver was inspected for bile leak and hemostasis and a drain was placed. The operation was performed using a low central venous pressure $(<5 \mathrm{~mm})$ and high intraabdominal pressure $(15 \mathrm{~mm} \mathrm{Hg})$ to decrease bleeding as in other hepatectomies.

Anatomical left lateral hepatectomy (Figure 3) : The cyst was isolated from rest of the abdominal cavity using povidone iodine soaked pads as in a cystopericystectomy. Dissection to the left of falciform ligament, carefully preserving the segment IV inflow, was done to isolate and divide the left lateral vascular pedicle draining segments II and III. After the vascular demarcation, a combination of $\mathrm{CUSA}^{\mathrm{TM}}$, Harmonic Scalpel $^{\mathrm{TM}}$, and electrocautery were used for parenchymal transection. The left hepatic vein was transected using either a stapler or vascular clips (Hem-o-lok clips $\left.{ }^{\circledR}\right)$. The specimen was put in an endo-bag and retrieved after extending the incision of the umbilical port and decompressing the cyst. The resection surface of the liver was inspected for bile leak and hemostasis and a drain was placed. 
Table 1: Details of patients undergoing laparoscopic radical surgery for hydatid cyst of liver

\begin{tabular}{|c|c|c|c|c|c|c|c|}
\hline & Age & Sex & Year & $\begin{array}{l}\text { Location } \\
\text { in segment }\end{array}$ & Size $(\mathrm{cm})$ & Surgery & $\begin{array}{l}\text { Conversion } \\
\text { to open }\end{array}$ \\
\hline 1 & 24 & M & 2006 & II/III & $7 \times 5$ & Left lateral hepatectomy & No \\
\hline 2 & 56 & $\mathrm{~F}$ & 2007 & IV & $6 \times 5$ & Cystopericystectomy & No \\
\hline 3 & 50 & $\mathrm{~F}$ & 2007 & VI/VII/VIII & $8 \times 6$ & Cystopericystectomy & Yes \\
\hline 4 & 44 & M & 2008 & VI/VII/VIII & $7.5 \times 6$ & Cystopericystectomy & Yes \\
\hline 5 & 25 & $\mathrm{~F}$ & 2008 & II/III & $6 \times 6$ & Left lateral hepatectomy & Yes \\
\hline 6 & 24 & $\mathrm{~F}$ & 2009 & IV/VIII & $6 \times 6$ & Near total cystopericystectomy & Yes \\
\hline 7 & 40 & $\mathrm{~F}$ & 2009 & IV/VI/VII/VIII & $10 \times 9.5$ & Cystopericystectomy & Yes \\
\hline 8 & 32 & $\mathrm{~F}$ & 2009 & VI/VII & $10 \times 6.4$ & Cystopericystectomy & No \\
\hline 9 & 44 & $\mathrm{~F}$ & 2009 & II/III & $8 \times 6$ & Left lateral hepatectomy & No \\
\hline 10 & 25 & $\mathrm{~F}$ & 2009 & $\mathrm{~V} / \mathrm{VI}$ & $3 \times 3$ & Cystopericystectomy & No \\
\hline 11 & 32 & $\mathrm{~F}$ & 2010 & VI & $8 \times 6$ & Cystopericystectomy & No \\
\hline 12 & 40 & M & 2011 & IV/VIII & $5 \times 5$ & Cystopericystectomy & No \\
\hline 13 & 23 & $\mathrm{~F}$ & 2011 & $\mathrm{II} / \mathrm{III}$ & $9 \times 8$ & Left lateral hepatectomy & No \\
\hline 14 & 19 & $\mathrm{~F}$ & 2012 & II/III & $12 \times 10$ & Left lateral hepatectomy & No \\
\hline
\end{tabular}

\section{Results}

During the period between January 2006 and December 2011, 56 patients with hepatic hydatid cysts were managed surgically at our center. Of these, 35 were treated with resectional surgery and 21 underwent a surgical drainage. Of the 35 patients who underwent a resectional procedure, a total of 14 patients were planned for a laparoscopic resection. There were 11 females and 3 males. The mean age was 34 years (Range: 19- 56 years). Abdominal pain was the most common presenting complaint in 13 patients. One patient presented with jaundice. This patient had a cysto-biliary communication and underwent preoperative biliary stenting. In 6 patients the cyst was located in the left lobe, 5 patients had the cyst in the right lobe and in 3 patients, cysts were present in both the lobes. Four patients had Gharbi Type I cysts, 4 had Type II cysts and 6 patients had Gharbi Type III cysts. The size of the cyst ranged between $3 \times 3 \mathrm{~cm}$ to 10 $x 9.5 \mathrm{~cm}$. Two patients had failed a prior PAIR procedure (due to aspiration of thick pultaceous material in one and cysto-biliary communication in another).

A laparoscopic resectional surgery could be accomplished in 9 of the 14 patients (Table 1). In 5 patients a laparoscopic cystopericystectomy was done and in 4 a laparoscopic left lateral hepatectomy was done. The 
mean intra-operative blood loss was $111.1 \mathrm{~mL}$ (Range: 50-250 $\mathrm{mL}$ ) and the mean operating time was $243.3 \mathrm{~min}$ (Range: 180-300min). There was no spillage of the cyst contents in any of the patient. All patients were allowed orally on the first postoperative day. None of the patients had any bile leak and the abdominal drain was removed in all on the third postoperative day. There were no wound infections and none of the patients developed any cavityrelated complications. At a median follow-up 54 (Range: 21-86) months, none of the patients had evidence of local or extrahepatic recurrence and have excellent cosmetic results.

In five patients, the laparoscopic procedure was abandoned and converted to an open operation. The reasons for conversion were either inability to complete the resection laparoscopically or bleeding. All these patients underwent a resectional procedure after conversion to open. In 3 of the 5 patients in whom laparoscopic resection could not be completed, the cysts were located in Segments VI/VII/VIII/IVA. There were no conversions from the latter half of 2009 i.e. there were conversions for posteriorly placed cysts in the early part of our experience.

\section{Discussion}

Most hydatid cysts are asymptomatic and are discovered incidentally. When symptomatic, they often present with abdominal pain, fullness and rarely with complications like jaundice, infection, and rupture. Palliation of symptoms and prevention of complications are the main rationale for the treatment of Echinococcal liver disease. ${ }^{8}$ To achieve this, most hydatid cysts require some form of management that may be pharmacotherapy, a percutaneous radiological intervention or surgery. The role of surgery is being redefined in the context of emerging non-surgical techniques like PAIR and PAIR-D; however, it still remains the gold standard for managing hydatid cysts of the liver. ${ }^{9}$

The goal of any form of surgical treatment for hydatid disease is inactivation and complete removal of all viable scolices and germinal membranes and management of the residual cavity with minimal morbidity and mortality. The management depends on the condition of the patient, nature of the cyst, number, size, location, the presence of cysto-biliary communication and the available expertise. The various surgical options can be broadly grouped into either conservative or radical. Radical surgery involves removal of the complete cyst with or without adjacent liver parenchyma (cystopericystectomy, formal hepatectomy). The cyst is not opened and thus spillage of contents is avoided. Such an approach has several advantages such as reduced local and distant recurrence, better identification and management of cysto-biliary communications, decreased incidence of biliary fistulas and cavity-related complications, shorter hospital stay, reduced morbidity and mortality. It also reduces the need for postoperative drug therapy. ${ }^{3,4}$ Despite these, a cyst in difficult location may pose a surgical challenge and increases the risk of surgical morbidity. In such situations, and considering the benign nature of the disease, it may be worthwhile to resort to conservative surgery. Cysts located close to major vascular pedicles like portal vein, hepatic vein or IVC, and cysts in a cirrhotic liver may be considered for drainage or near-total cystopericystectomy.

The benefits of radical surgery are well established in the literature. In a large single-center study comparing radical with conservative surgery, the postoperative morbidity and recurrence were significantly lower in the radical group (3.2\% vs. $11.6 \%$ and $3.2 \%$ vs $24 \%) .{ }^{4}$ In another large series of 372 patients, patients who underwent a radical surgery had shorter hospital stay $(8.65$ vs. 14.9 days), morbidity (13.3 vs. $31.4 \%$, p value $<0.001$ ), and mortality ( 0 vs. $3.8 \%$, pvalue $<0.01$ ). The recurrence rate was $1.85 \%$ after radical surgery versus $11.9 \%$ in the conservative surgery group $(p<0.0001) .{ }^{3}$ While the literature supports resection as the treatment of choice for hydatid cysts, there has been a resurgence of drainage procedures especially with the advent of laparoscopic surgery. ${ }^{10-12}$ Although such an approach has the benefits of being minimally invasive, it suffers from the same limitations as open drainage operations as regards the cavity-related issues, management of cysto-biliary communications and chances of local and distant recurrence. While a laparoscopic drainage may be justified in patients wherein due to anatomical location of the cyst, a drainage operation is indicated, it should not be the preferred approach because of technical difficulty 
in performing a laparoscopic cystopericystectomy or a formal hepatectomy. In such cases, it would be preferable to perform an open cystopericystectomy rather than a laparoscopic drainage. Manterola et al described a laparoscopic cystopericystectomy in 8 patients, in whom the cysts were isolated, vacuumed/contents evacuated, and then a pericystectomy performed. ${ }^{13}$ While such a procedure would decrease the incidence of cavityrelated problems and may decrease the local recurrence rates, the risk of spillage with hydatid dissemination and anaphylaxis still remains.

Good patient selection and adequate training in laparoscopic surgery are important prior to contemplating a laparoscopic resectional procedure for hydatid cyst of the liver. Small, peripheral cysts located in the anterior segment are most suited for a laparoscopic resection. Cysts encroaching the porta hepatis and those close to major bilio-vascular pedicles should be managed with either open cystopericystectomy or a drainage operation. Laparoscopic resection for large posteriorly located cysts is technically challenging but may be accomplished with increasing experience.

The main objective in a laparoscopic resection of hydatid is to remove the cyst in its entirety, prevent any spillage of contents, and minimize blood loss. Although in a cystopericystectomy, the entire cyst with the adjacent normal liver parenchyma is resected, isolation of the cyst with scolicidal (betadine) soaked gauzes prior to dissection is important to prevent inadvertent spillage of contents in case of accidental cyst rupture during dissection. Dissection with CUSA $^{\mathrm{TM}}$ is especially useful while performing a laparoscopic cystopericystectomy. Cysts replacing an entire lobe are best managed by anatomical liver resections with an inflow and outflow control. Meticulous dissection through the liver parenchyma, low central venous pressure, and increased intra-abdominal pressure pneumoperitoneum result in minimal blood loss during the parenchymal transection. Following the completion of resection, the specimen is put into an endobag and brought to the surface of the skin. The cyst is then opened and all contents are evacuated. The cyst can then be removed via a small incision (extended port incision). It is important at the end of the procedure to ask the anesthetist to increase the CVP and decrease the intraabdominal pneumoperitoneum pressure to ensure adequate hemostasis.

In the present study, 14 patients were taken up for laparoscopic radical surgery for liver hydatid. A parenchyma preserving approach (cystopericystectomy) was the procedure of choice whenever feasible, and a formal hepatectomy was done only in cases wherein an entire hepatic lobe was replaced by the hydatid cyst. A complete laparoscopic resection was accomplished in 9 patients. The reasons for failure were bleeding and presence of extensive perihepatic adhesions. Most of the failures occurred in cysts located in the posterosuperior segment of the liver and in the early part of the learning curve. In the study by Misra et al, 2 of the 9 patients who underwent a laparoscopic cystopericystectomy required conversion to open owing to uncontrolled bleeding. ${ }^{14}$ In both these patients, the cysts were large in size (11 and 15 $\mathrm{cm}$ ) and one of them was posteriorly placed.

\section{Conclusions}

Laparoscopic resection for hydatid cyst of liver is a feasible option and should be considered in these patients. Wherever possible, a complete excision of the cyst should be performed, regardless of the approach (laparoscopic or open). Inability to perform a laparoscopic resectional procedure should not be used as an argument to perform a laparoscopic drainage. Laparoscopic management of hydatid cyst should be aimed at complete resection and minimal access approach should not be used as an advocate for drainage procedure.

\section{References}

1. Djuricic SM, Grebeldinger S, Kafka DI, Djan I, Vukadin M, Vasiljevic ZV. Cystic echinococcosis in children - the seventeen-year experience of two large medical centers in Serbia. Parasitol Int. 2010 Jun; 59(2): 257-61.

2. Morris D, Richards K. Hydatid disease. Oxford: Butterworth-Heinemann; 1992.

3. Priego P, Nuño J, López Hervás P. Hepatic hydatidosis. Radical vs. conservative surgery: 22 years of experience. Rev Esp Enferm Dig. 2008;100(2):82-5.

4. Aydin U, Yazici P, Onen Z. The optimal treatment of hydatid cyst of the liver: radical surgery with a significant reduced risk of recurrence. Turk J Gastroenterol. 2008; 
19(1): 33-9.

5. Gupta N, Javed A, Puri S, Jain S, Singh S, Agarwal AK. Hepatic hydatid: PAIR, drain or resect? J Gastrointest Surg. 2011 Oct;15(10):1829-36.

6. Gharbi HA, Hassine W, Brauner MW, Dupuch K. Ultrasound examination of the hydatic liver. Radiology 1981; 139(2): $459-63$.

7. Guidelines for treatment of cystic and alveolar echinococcosis in humans. WHO

Informal Working Group on Echinococcosis. Bull World Health Organ. 1996; 74(3): 231-42.

8. Buttenschoen K, Carli Buttenschoen D. Echinococcus granulosus infection: the challenge of surgical treatment. Langenbecks Arch Surg. 2003 Sep; 388(4): 218-30.

9. Yorganci K, Sayek I. Surgical treatment of hydatid cysts of the liver in the era of percutaneous treatment. Am J Surg
2002; 184:63; 9 .

10. Acarli K. Controversies in the laparoscopic treatment of hepatic hydatid disease. HPB (Oxford). 2004;6(4):213-21.

11. Chowbey PK, Shah S, Khullar R. Minimal access surgery for hydatid cyst disease: laparoscopic, thoracoscopic, and retroperitoneoscopic approach. J Laparoendosc Adv Surg Tech A. 2003; 13(3):159-65.

12. Palanivelu C, Jani K, Malladi V. Laparoscopic management of hepatic hydatid disease. JSLS. 2006; 10(1):56-62.

13 Manterola C, Fernández O, Muñoz S, Vial M, Losada H, Carrasco R, Bello N, Barroso M. Laparoscopic pericystectomy for liver hydatid cysts. Surg Endosc. 2002 Mar;16(3):521-4

14. Misra MC, Khan RN, Bansal VK. Laparoscopic pericystectomy for hydatid cyst of the liver. Surg Laparose Endosc Percutan Tech. 2010; 20(1): 24-6. 